\title{
Second-look surgery following Hartmann's procedure for obstructive left-sided colorectal cancer
}

\author{
NAOHITO BEPPU $^{1}$, FUMIHIKO KIMURA ${ }^{1}$, NAGAHIDE MATSUBARA ${ }^{2}$, MASASHI NODA $^{2}$, \\ NAOHIRO TOMITA $^{2}$, HIDENORI YANAGI $^{1}$ and NAOKI YAMANAKA ${ }^{1}$ \\ ${ }^{1}$ Department of Surgery, Meiwa Hospital, Nishinomiya, Hyogo 663-8186; \\ ${ }^{2}$ Department of Surgery, Hyogo College of Medicine, Nishinomiya, Hyogo 663-8501, Japan
}

Received December 9, 2014; Accepted January 18, 2016

DOI: $10.3892 / 01.2016 .5084$

\begin{abstract}
The aim of the present study was to investigate the short- and long-term outcomes of patients undergoing second-look surgery following Hartmann's procedure for obstructive left-sided colorectal cancer (LSCC). All patients included in the present study had undergone radical surgery with Hartmann's procedure for obstructive LSCC. Adjuvant chemotherapy was recommended for all patients, and patients with no signs of recurrence following six months of surveillance were planned to undergo second-look surgery. The aim of second-look surgery was early detection of local recurrence and determination of the efficacy of laparoscopic Hartmann procedure reversal. A total of 15 patients with locally advanced colorectal cancer were included in the study. Three patients exhibited peritoneal dissemination at the time of laparoscopic Hartmann procedure reversal and underwent partial peritonectomy. Following adjuvant chemotherapy treatment, laparoscopic Hartmann procedure reversal was performed in all patients. However, two patients underwent colo-anal anastomosis, and two patients underwent right-sided colon or ileum reconstruction. Regarding the oncological outcomes, two of three patients in whom peritoneal dissemination was identified during laparoscopic Hartmann procedure reversal were eventually in remission following the initial surgery and the second-look surgery with partial peritonectomy. Favorable long-term outcomes were observed in 12/15 patients due to no recurrence, which may be due to the surgical techniques used and the timing of the second-look surgery following Hartmann's procedure for the treatment of obstructive LSCC.
\end{abstract}

Correspondence to: Dr Naohito Beppu, Department of Surgery, Meiwa Hospital, 4-31 Agenaruo-cho, Nishinomiya, Hyogo 663-8186, Japan

E-mail: beppu-n@hyo-med.ac.jp

Key words: obstructive left-sided colorectal cancer, second-look surgery, Hartmann's procedure, adjuvant chemotherapy, peritoneal dissemination

\section{Introduction}

Approximately $15-20 \%$ of patients with left-sided colorectal cancer (LSCC) present with symptoms of acute obstruction (1-4). Patients with obstructive LSCC require emergency surgery, and a high rate of surgical complications and poor prognosis have been reported in these cases $(5,6)$. Emergency management of acute left-sided colonic obstruction remains controversial and at present several treatments options exist, including simple colostomy, primary resection with end colostomy (Hartmann's procedure), one-stage resection anastomosis (subtotal or segmental colectomy) with or without intraoperative colonic irrigation and colonic stenting (7). Emergency surgery for acute colonic obstruction carries a significant risk of mortality and morbidity: The 30-day postoperative mortality rate is $7.6 \%$, with anastomotic dehiscence occurring in $4.9 \%$ of cases (2). Thus, $46 \%$ of patients require stoma treatment that is permanent (2). To reduce the risk of complications, laparoscopic Hartmann procedure reversal is commonly used for LSCC, since it is considered to reduce the morbidity caused by anastomotic dehiscence (8). However, re-establishing intestinal continuity during laparoscopic Hartmann procedure reversal for LSCC remains a major problem and it carries a serious risk of significant morbidity, with reported anastomotic leak rates of 4-16\% and a mortality rate of up to $10 \%(9,10)$. Stoma closure is difficult, as colorectal malignancies require segmental colostomy with lymph node dissection, which results in a high rate of dense adhesions (10). Additionally, LSCC is associated with a poor prognosis due to the large size and high frequency of gastrointestinal strictures (11). In addition, certain cases exhibit peritoneal dissemination, which exposes the cancer cells to the serosa (12). Thus, it is important to consider these surgical and oncological factors when planning a laparoscopic Hartmann procedure reversal for LSCC, since these difficulties may prevent the success of this surgical procedure, which was previously reported to be $20 \%$ for LSCC (11).

In the present study, second-look surgery was performed in patients with no signs of recurrence following six months of surveillance subsequent to Hartmann's procedure. The present study aimed to determine the efficacy of laparoscopic Hartmann procedure reversal and to assess local recurrence. The aim of the present study was to investigate the short- and 
long-term outcomes of second-look surgery following Hartmann's procedure for LSCC.

\section{Materials and methods}

Patients. The present study was retrospective, however, patient information was collected prospectively. The study analyzed patients that underwent laparoscopic Hartmann procedure reversal for LSCC between January 2007 and December 2013, at Meiwa Hospital (Nishinomiya, Japan). The inclusion criteria for patients were as follows: i) Hartmann's procedure was performed within $48 \mathrm{~h}$ of admission to hospital due to LSCC; ii) diagnosis of colorectal carcinoma was confirmed upon pathological examination of the surgical specimen; and iii) radical surgery was performed during the initial surgery. Therefore, patients with benign disease, such as diverticulosis, and patients with distant, liver or local residual disease at the time of initial surgery were excluded from the study.

A total of 43 patients underwent Hartmann's procedure due to LSCC at Meiwa Hospital. A total of 25 patients underwent radical surgery, and 18 patients exhibited residual disease (local, distant or liver metastasis). All 25 patients who underwent radical surgery were confirmed to exhibit no signs of recurrence following 6 months of surveillance. However, 10 patients were unsuitable for second-look surgery due to advanced age (>80 years) or high American Society of Anesthesiologists (ASA) score (ASA $>$ II) (13). Therefore, 15 patients were included in the present study. Written informed consent was obtained from all patients and the study was approved by the ethics committee of Meiwa Hospital.

Initial surgery. During Hartmann's procedure, radical surgery with lymph node dissection was conducted, and the vascular pedicle was transected at the inferior mesenteric artery, followed by high-tie ligation. Resected specimens of colorectal malignancies were removed to leave the proximal segment of the bowel as an end colostomy, while the remaining distal bowel was oversewn as a rectal stump. The resection line of the distal bowel was generally located at the level of the peritoneal reflection with consideration of the blood supply, and the distal rectal stump was marked with non-absorbable PROLENE ${ }^{\circledR}$ sutures (Ethicon, Inc., Summerville, NJ, USA). Following surgery, the administration of adjuvant oral 5-fluorouracil (5-FU)-based chemotherapy (TS-1 ${ }^{\circledR}$; Taiho Pharmaceutical Co, Ltd., Tokyo, Japan; $80 \mathrm{mg} / \mathrm{m}^{2} /$ day) was recommended for all patients, and patients with $\mathrm{N} 2$ stage or a positive pathological $\mathrm{P}$ stage also received oxaliplatin (Elplat ${ }^{\circledR}$, Yakult Pharmaceutical Co, Ltd., Tokyo, Japan; 85 mg/m²/day) intravenously as additional adjuvant chemotherapy for a total of 6 months.

Second-look surgery. Following six months of surveillance, second-look surgery was considered in cases that exhibited no signs of recurrence during chest and abdominal computed tomography (CT; SOMATOM Definition Flash; Siemens AG, Munich, Germany) examinations. During the second-look surgery, patients were assessed for local recurrence, and the intestinal continuity, which was re-established during laparoscopic Hartmann procedure reversal, was reviewed. If localized recurrence or peritoneal dissemination was observed, the carcinomatous lesion was resected and anastomosis was then performed.

Patient surveillance. Following second-look surgery, patient surveillance was performed as follows: Chest and abdominal CT examinations were conducted every six months; colonoscopy was performed annually; and blood tests, which included measurement of carcinoembryonic antigen and cancer antigen 19-9 levels, were performed every three months. Local recurrence was defined as the identification of a recurrent tumor within the pelvis, while systemic recurrence was defined as the presence of recurrent disease outside the pelvis.

The pathological findings of the resected specimens were assessed according to the Japanese General Rules for Clinical and Pathological Studies on Cancer of the Colon, Rectum and Anus (7th edition) (14).

Statistical analysis. Time to recurrence was measured from the date of second-look surgery to the date of recurrence, and the long-term outcomes were assessed to determine the 3-year local relapse-free survival (LFS) and recurrence-free survival (RFS) rates, which were estimated using the Kaplan-Meier method. All data analysis was performed using JMP 10.0 statistical software (SAS Institute Inc., Cary, NC, USA).

\section{Results}

Patients' characteristics at the time of initial surgery. The patient cohort included 10 females (66.7\%) and 5 males (33.3\%), with a median age of 61 years (range, $32-75$ years) (Table I). All patients exhibited an ASA score of I or II, and the median body mass index was $21 \mathrm{~kg} / \mathrm{m}^{2}$ (range, $16-31 \mathrm{~kg} / \mathrm{m}^{2}$ ). The most common type of tumor was rectosigmoid carcinoma (10 patients; $66.7 \%$ ). A total of 14 patients exhibited pathological stage T3 disease, whereas 1 patient exhibited stage T4 (sigmoid) disease. In addition, 8 patients exhibited lymph node metastasis (Table I). At laparotomy, 3 patients were observed to exhibit peritoneal dissemination (Table I); however, all lesions were localized and were radically resected. Following surgery, all patients received adjuvant chemotherapy, and 4 patients received oxaliplatin as additional adjuvant chemotherapy.

Patients' characteristics at the time of second-look surgery. Following six months of surveillance, none of the patients exhibited signs of recurrence on physical or radiological examination. The median interval between initial and second-look surgery was 273 days (range, 212-592 days; data not shown). During the second-look surgery, 11 patients underwent colorectal anastomosis (CRA), 2 patients underwent colo-anal anastomosis (CAA), 1 patient underwent ceco-rectal anastomosis (Ce-RA) and 1 patient underwent ileo-anal canal anastomosis (IACA) (Table II). CRA has been proposed as the standard procedure to re-establish intestinal continuity (10-12). However, 2 patients underwent CAA due to the formation of dense adhesions between the rectal stump and bladder, small bowel and vagina. In addition, 2 patients with short-reconstructed colons underwent further mobilization of the splenic flexure to achieve sufficient mobility of the descending colon, and therefore, were reconstructed with 
Table I. Patients' characteristics at the time of initial surgery.

\begin{tabular}{|c|c|}
\hline Parameter & $\mathrm{n}$ \\
\hline \multicolumn{2}{|l|}{ Gender } \\
\hline Male & 5 \\
\hline Female & 10 \\
\hline \multicolumn{2}{|l|}{ Age, years } \\
\hline Median (range) & $61(32-75)$ \\
\hline \multicolumn{2}{|l|}{$\mathrm{BMI}, \mathrm{kg} / \mathrm{m}^{2}$} \\
\hline Median (range) & $21(16-31)$ \\
\hline \multicolumn{2}{|l|}{ ASA score } \\
\hline I & 13 \\
\hline II & 2 \\
\hline III & 0 \\
\hline IV & 0 \\
\hline \multicolumn{2}{|l|}{ Tumor location } \\
\hline Descending & 2 \\
\hline Sigmoid & 3 \\
\hline Rectosigmoid & 10 \\
\hline \multicolumn{2}{|l|}{ Pathological T stage $^{\mathrm{a}}$} \\
\hline $\mathrm{T} 0-2$ & 0 \\
\hline $\mathrm{T} 3$ & 14 \\
\hline $\mathrm{T} 4$ & $1^{\mathrm{c}}$ \\
\hline \multicolumn{2}{|c|}{ Pathological N stage $^{\mathrm{a}}$} \\
\hline $\mathrm{N}^{-}$ & 7 \\
\hline N1 & 6 \\
\hline $\mathrm{N} 2$ & 2 \\
\hline \multicolumn{2}{|l|}{ Pathological P stage ${ }^{a}$} \\
\hline $\mathrm{P}^{-}$ & 12 \\
\hline $\mathrm{P}^{+}$ & 3 \\
\hline \multicolumn{2}{|l|}{ TNM stage ${ }^{\mathrm{b}}$} \\
\hline II & 5 \\
\hline III & 7 \\
\hline IV & 3 \\
\hline \multicolumn{2}{|c|}{ Adjuvant chemotherapy } \\
\hline Oral intake only & 11 \\
\hline Oxaliplatin added & 4 \\
\hline
\end{tabular}

${ }^{a}$ According to the Japanese Clinical and Pathological Guidelines for the Colon, Rectum and Anus (7th edition) (14). ${ }^{\mathrm{b}}$ According to the National Comprehensive Cancer Network Clinical Practice Guidelines in Oncology (15). 'Sigmoid. BMI, body mass index; ASA, American Society of Anesthesiologists; TNM, tumor-nodemetastasis.

the right-side colon or ileum. A total of 5 patients required a diverting ileostomy due to incomplete anastomosis.

Second-look surgery revealed that $2 / 3$ patients with peritoneal dissemination at the time of initial surgery exhibited localized peritoneal dissemination at the time of second-look surgery. However, these patients underwent radical surgery with partial peritonectomy. The other patient did not exhibit peritoneal dissemination at the time of second-look surgery, and exhibited no signs of local or distant recurrence following

Table II. Patients' characteristics at the time of second-look surgery.

Parameter

$\mathrm{n}$

Surgical procedure

CRA 11

CAA 2

Ce-RA

IACA

1

1

Diverting ileostomy

Yes 5

No

Length of surgery, min

Median (range)

$257(189-600)$

Amount of blood lost, $\mathrm{ml}$

Median (range)

$405(290-3,340)$

Local recurrence

Yes

$2^{\mathrm{a}}$

13

Distant recurrence

Yes

0

No

TNM residual tumor classification ${ }^{\mathrm{b}}$

R0

$\mathrm{R} 1-2$

0

Complications

Wound infection $\quad 2$

Ileus $\quad 2$

Pelvic infection $\quad 1$

Abdominal hernia $\quad 1$

Intraoperative injury (bladder) $\quad 1$

Wound infection and ileus $\quad 1$

Wound infection and pelvic infection $\quad 1$

Wound infection and cholecystitis $\quad 1$

None $\quad 5$

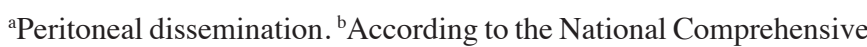
Cancer Network Clinical Practice Guidelines in Oncology (15). CRA, colorectal anastomosis; CAA, colo-anal anastomosis; Ce-RA, ceco-rectal anastomosis; IACA, ileo-anal canal anastomosis; R0, no residual tumor confirmed microscopically; R1, microscopic tumor residue; R2, macroscopic tumor residue.

37 months of follow-up. In certain cases, postoperative complications developed, including wound infections $(n=5)$, ileus $(n=3)$, pelvic infection $(n=2)$, cholecystitis $(n=1)$ abdominal hernia $(n=1)$ and intraoperative bladder injury $(n=1$; Table II); however, all patients were successfully treated conservatively using infusion fluids and/or antibiotics, with the exception of the abdominal hernia patient, who remains under observation.

Long-term outcomes following second-look surgery. The median follow-up period subsequent to second-look surgery was 35.4 months (range, 9.0-64.0 months; data not shown). 
The 3-year LFS and RFS rates were 92.3 and $78.6 \%$, respectively (Fig. 1). Of the 2 patients who exhibited peritoneal dissemination at the time of second-look surgery, 1 patient was recurrence-free following 27 months of follow-up, and 1 patient exhibited local recurrence in the peritoneum after 30 months. Of the 5 patients who required diverting ileostomy, the stoma in 4 patients had already closed, and the stoma in the remaining patient was planned to be closed. Therefore, intestinal continuity was considered to have been maintained in all patients 8 months subsequent to the performance of second-look surgery.

\section{Discussion}

Laparoscopic Hartmann procedure reversal for LSCC is associated with a substantial risk of surgical complications, as the surgery may result in a short rectal stump, dense adhesions and a short left-side reconstructed colon (10-12). In addition, local recurrence, in particular peritoneal dissemination, disrupts intestinal continuity (10-12). However, patients who are able to undergo stoma closure experience an improved quality of life following surgery (10-12).

Two major problems are associated with the technique used for laparoscopic Hartmann procedure reversal exist: As a result of the procedure, dense adhesions develop between the rectal stump and other tissues, and the left-side reconstructed colon may be of an inadequate length to reach to the rectal stump $(16,17)$. In the present study, a high-tie ligation was routinely performed, and consequently, the distal rectal stump was transected at the level of the peritoneal reflection following consideration of the blood supply. Previous studies have reported that the length of the distal rectal stump is associated with postoperative complications, and that a short distal rectal stump contributes to a longer duration of surgery and increased postoperative complications $(16,17)$. Although the distal rectal stump may be marked with non-absorbable sutures during the initial surgery, it may be difficult to identify the rectal stump due to the development of dense adhesions with the bladder or vagina, in males and females, respectively (18). In certain cases, marking sutures cannot be identified and thus, rectal dilators or a sigmoidoscope may be used, as well as insufflating the bladder, which enables the safe dissection and localization of the rectal stump $(10,19)$. However, despite using these approaches in the present study, intraoperative complications occurred in 2 patients due to dense adhesions. In the present study, intestinal continuity was maintained in all patients following CAA. However, the adhesions led to an inability to mobilize the rectum, and the CAA technique was a useful method to prevent a failed attempt of maintaining intestinal continuity in cases with severe adhesions.

Additionally, surgery may result in the left-sided reconstructed colon being of inadequate length, leading to a subsequent inability to reach to the rectal stump $(16,17)$. In the present study, this occurred in 2 patients, and sub-total or total colectomy with Ce-RA or IACA were selected to use the right-sided colon or ileum, which released the excessive tension on the anastomosis, thus avoiding associated complications.

With regard to the timing of the laparoscopic Hartmann procedure reversal, Pearce et al (20) reported that postoperative complications were significantly greater in patients who underwent surgery within six months of the prodedure compared with those who underwent surgery more

\section{- - Local relapse-free survival} Recurrence-free survival

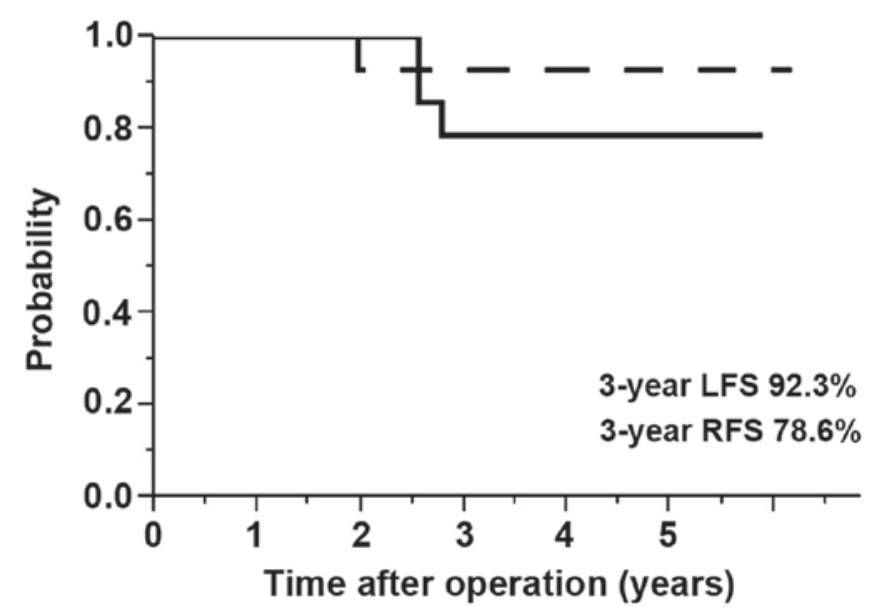

Figure 1. Long-term outcomes following second-look surgery. Time to recurrence was measured from the time of second-look surgery. LFS, local relapse-free survival; RFS, recurrence-free survival.

than six months subsequent to the procedure. By contrast, Fleming et al (21) reported that a longer interval between initial surgery and laparoscopic Hartmann procedure reversal ( $>9$ months) was associated with a higher risk of postoperative complications. However, these reports were assessed according to the risk of postoperative morbidity, and analyzed only in patients with benign disease $(21,22)$. By contrast, the present study only analyzed patients with colorectal malignancies.

Time to progression (TTP) is defined as the time period between the start of disease treatment and disease progression (23). Previous studies have demonstrated that chemotherapy reduces the postoperative recurrence rate, and TTP is one of the factors used to evaluate the effects of chemotherapy $(24,25)$. The TTP following treatment with oral tegafur/uracil with leucovorin (LV), 5-FU/LV and capecitabine has been reported to be 3.5, 3.8 and 4.3 months, respectively $(24,25)$. Therefore, exceeding these periods may aid early detection of tumor recurrence $(22,24)$. Therefore, in the present study, second-look surgery was performed six months subsequent to the initial surgery, and aimed to assess the patients for local recurrence and to review the efficacy of the laparoscopic Hartmann procedure reversal.

In the current study, 3 patients exhibited peritoneal dissemination at the time of initial surgery, 2 of which appear to have been in remission at present. In $\sim 10 \%$ of colorectal cancer cases, patients present with peritoneal dissemination at the time of diagnosis, and $25 \%$ of patients develop peritoneal dissemination at recurrence (25-28). At present, no evidence suggests that performing peritonectomy for peritoneal dissemination of colorectal malignancies improves patients' outcomes; however, partial peritonectomy for localized peritoneal dissemination may improve patients' survival (29). Therefore, the early detection of peritoneal dissemination and prompt treatment may have contributed to the favorable outcomes observed in the present cases.

The current study presents certain limitations. Firstly, the present study is a single arm of a retrospective study, and 
the patient cohort was small. Secondly, the indications for Hartmann's procedure have decreased in the past years, as other surgical procedures for LSCC are currently available, including primary anastomosis with ileostomy or elective surgery using self-expanding metallic stents (30). However, perforated or pre-perforated LSCC remain reasonable indications for Hartmann's procedure (30).

In conclusion, hand-sewn CAA and reconstruction with right-sided colon or ileum may be useful to prevent failed attempts to maintain intestinal continuity in patients with LSCC. With regard to the oncological outcomes, TTP following oral adjuvant chemotherapy is generally $<6$ months; therefore, any time subsequent to this period would be reasonable to conduct a second-look surgery to confirm whether recurrence has occurred. Furthermore, early detection and intensive treatment for localized peritoneal dissemination may lead to advances in colorectal cancer treatment in certain cases.

\section{Acknowledgements}

The authors would like to thank Dr Kakuno (Department of Pathology, Meiwa Hospital, Nishinomiya, Japan) for performing the pathological assessments.

\section{References}

1. Phillips RK, Hittinger R, Fry JS and Fielding LP: Malignant large bowel obstruction. Br J Surg 72: 296-302, 1985.

2. Mella J, Biffin A, Radcliffe AG, Stamatakis JD and Steele RJ: Population-based audit of colorectal cancer management in two UK health regions. Colorectal Cancer Working Group, Royal College of Surgeons of England Clinical Epidemiology and Audit Unit. Br J Surg 84: 1731-1736, 1997.

3. Serpell JW, McDermott FT, Katrivessis H and Hughes ES: Obstructing carcinomas of the colon. Br J Surg 76: 965-969, 1989.

4. Umpleby HC and Williamson RC: Survival in acute obstructing colorectal carcinoma. Dis Colon Rectum 27: 299-304, 1984.

5. Finan PJ, Campbell S, Verma R, MacFie J, Gatt M, Parker MC, Bhardwaj R and Hall NR: The management of malignant large bowel obstruction: ACPGBI position statement. Colorectal Dis 9 (Suppl 4): 1-17, 2007.

6. McArdle CS and Hole DJ: Emergency presentation of colorectal cancer is associated with poor 5-year survival. Br J Surg 91: 605-609, 2004.

7. Trompetas V: Emergency management of malignant acute left-sided colonic obstruction. Ann R Coll Surg Engl 90 181-186, 2008

8. Borowski DW, Kanakala V, Agarwal AK, Tabaqchali MA, Garg DK and Gill TS: Single-port access laparoscopic reversal of Hartmann operation. Dis Colon Rectum 54: 1053-1056, 2011.

9. Breitenstein S, Rickenbacher A, Berdajs D, Puhan M, Clavien PA and Demartines N: Systematic evaluation of surgical strategies for acute malignant left-sided colonic obstruction. Br J Surg 94: 1451-1460, 2007.

10. Rosen MJ, Cobb WS, Kercher KW, Sing RF and Heniford BT: Laparoscopic restoration of intestinal continuity after Hartmann's procedure. Am J Surg 189: 670-674, 2005.

11. Kristiansen VB, Lausen IM, Frederiksen HJ and Kjaergaard J: Hartmann's procedure in the treatment of acute obstructive left-sided colonic cancer. Ugeskr Laeger 155: 3816-3818, 1993 (In Danish).
12. Canney AL, Kevans D, Wang LM, Hyland JM, Mulcahy HE, O'Donoghue DP, O'Sullivan J, Geraghty R and Sheahan K: Stage II colonic adenocarcinoma: A detailed study of pT4N0 with emphasis on peritoneal involvement and the role of tumour budding. Histopathology 61: 488-496, 2012.

13. Saklad M: Grading of patients for surgical procedures. Anesthesiology 2: 281-284, 1941.

14. Japanese Society for Cancer of the Colon and Rectum: General rules for clinical and pathological studies on cancer of the colon, rectum, and anus. 7th edition. Kanehara Shuppan, Tokyo, Japan, 2006.

15. Engstrom PF, Arnoletti JP, Benson AB III, Chen YJ, Choti MA, Cooper HS, Covey A, Dilawari RA, Early DS, Enzinger PC, et al; National Comprehensive Cancer Network: NCCN Clinical Practice Guidelines in Oncology: Rectal cancer. J Natl Compr Canc Netw 7: 838-881, 2009.

16. Alberts JC, Parvaiz A and Moran BJ: Predicting risk and diminishing the consequences of anastomotic dehiscence following rectal resection. Colorectal Dis 5: 478-482, 2003.

17. Mäkelä JT, Kiviniemi H and Laitinen S: Risk factors for anastomotic leakage after left-sided colorectal resection with rectal anastomosis. Dis Colon Rectum 46: 653-660, 2003.

18. Oomen JL, Cuesta MA and Engel AF: Reversal of Hartmann's procedure after surgery for complications of diverticular disease of the sigmoid colon is safe and possible in most patients. Dig Surg 22: 419-425, 2005.

19. Anderson CA, Fowler DL, White S and Wintz N: Laparoscopic colostomy closure. Surg Laparosc Endosc 3: 69-72, 1993.

20. Pearce NW, Scott SD and Karran SJ: Timing and method of reversal of Hartmann's procedure. Br J Surg 79: 839-841, 1992.

21. Fleming FJ and Gillen P: Reversal of Hartmann's procedure following acute diverticulitis: Is timing everything? Int J Colorectal Dis 24: 1219-1225, 2009.

22. Douillard JY, Hoff PM, Skillings JR, Eisenberg P, Davidson N, Harper P, Vincent MD, Lembersky BC, Thompson S, Maniero A and Benner SE: Multicenter phase III study of uracil/tegafur and oral leucovorin versus fluorouracil and leucovorin in patients with previously untreated metastatic colorectal cancer. J Clin Oncol 20: 3605-3616, 2002.

23. Middleton G, Brown S, Lowe C, Maughan T, Gwyther S, Oliver A, Richman S, Blake D, Napp V, Marshall H, et al: A randomised phase III trial of the pharmacokinetic biomodulation of irinotecan using oral ciclosporin in advanced colorectal cancer: results of the Panitumumab, Irinotecan \& Ciclosporin in COLOrectal cancer therapy trial (PICCOLO). Eur J Cancer 49: 3507-3516, 2013.

24. Hoff PM, Ansari R, Batist G, Cox J, Kocha W, Kuperminc M, Maroun J, Walde D, Weaver C, Harrison E, et al: Comparison of oral capecitabine versus intravenous fluorouracil plus leucovorin as first-line treatment in 605 patients with metastatic colorectal cancer: Results of a randomized phase III study. J Clin Oncol 19: 2282-2292, 2001.

25. Verwaal VJ, van Ruth S, Witkamp A, Boot H, van Slooten G and Zoetmulder FA: Long-term survival of peritoneal carcinomatosis of colorectal origin. Ann Surg Oncol 12: 65-71, 2005.

26. Carraro PG, Segala M, Cesana BM and Tiberio G: Obstructing colonic cancer: Failure and survival patterns over a ten-year follow-up after one-stage curative surgery. Dis Colon Rectum 44: 243-250, 2001.

27. Jayne DG, Fook S, Loi C and Seow-Choen F: Peritoneal carcinomatosis from colorectal cancer. Br J Surg 89: 1545-1550, 2002.

28. Russell AH, Pelton J, Reheis CE, Wisbeck WM, Tong DY and Dawson LE: Adenocarcinoma of the colon: An autopsy study with implications for new therapeutic strategies. Cancer 56: 1446-1451, 1985.

29. Pestieau SR and Sugarbaker PH: Treatment of primary colon cancer with peritoneal carcinomatosis: Comparison of concomitant vs. delayed management. Dis Colon Rectum 43: 1341-1348, 2000.

30. Garber A, Hyman N and Osler T: Complications of Hartmann takedown in a decade of preferred primary anastomosis. Am J Surg 207: 60-64, 2014. 